\title{
Microbial investigations in throat swab and tracheal aspirate specimens are beneficial to predict the corresponding endotracheal tube biofilm flora among intubated neonates with ventilator-associated pneumonia
}

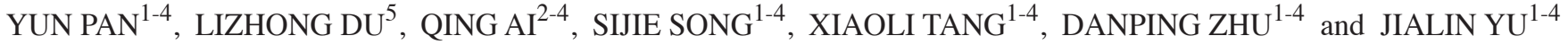 \\ ${ }^{1}$ Department of Neonatology, Children's Hospital of Chongqing Medical University, Chongqing 400014; \\ ${ }^{2}$ Ministry of Education Key Laboratory of Child Development and Disorders; ${ }^{3}$ Key Laboratory of Pediatrics in Chongqing, \\ Chongqing Medical University; ${ }^{4}$ Chongqing International Science and Technology Cooperation Center for Child \\ Development and Disorders, Children's Hospital of Chongqing Medical University, Chongqing 400014; ${ }^{5}$ Department of \\ Pediatrics, Children's Hospital of Zhejiang University School of Medicine, Hangzhou, Zhejiang 310003, P.R. China
}

Received October 11, 2015; Accepted February 14, 2017

DOI: $10.3892 / \mathrm{etm} .2017 .4631$

\begin{abstract}
Ventilator-associated pneumonia (VAP) is a common nosocomial infection in neonatal intensive care units with high morbidity and mortality. Bacterial biofilm in the endotracheal tube (ET) provides a notable and persistent source of pathogens that may cause VAP, and thus is important for VAP detection. However, during intubation microbial investigations in ET, samples are unavailable due to the infeasibility of collecting ET samples during intubation of neonates. It is therefore of great importance to find alternative sources of samples that can help identify the ET biofilm flora. In the present study, the microbial signatures of throat swabs and tracheal aspirates were compared with ET biofilm samples from VAP neonates using 16S ribosomal RNA gene polymerase chain reaction, denaturing gradient gel electrophoresis (DGGE), cloning and sequencing. Sequences were assigned to phylogenetic species using BLAST. Microbial diversity and richness among the three types of specimens were compared based on their DGGE fingerprints, and taxonomic characteristics based on the BLAST results. The microbial richness and diversity of ET biofilms were similar to tracheal aspirate
\end{abstract}

Correspondence to: Dr Jialin Yu, Department of Neonatology, Children's Hospital of Chongqing Medical University, 136 The Second Zhongshan Road, Yuzhong, Chongqing 400014, P.R. China E-mail: yujialin486486@hotmail.com

Abbreviations: DGGE, denaturing gradient gel electrophoresis; ET, endotracheal tube; NICU, neonatal intensive care unit; PICU, Pediatric intensive care unit; UPGMA, unweighted pair group method with arithmetic averages; VAP, ventilator-associated pneumonia

Key words: ventilated-associated pneumonia, endotracheal tube biofilm, throat swab, tracheal aspirate, microflora, intubated neonate yet significantly different from throat swab samples $(\mathrm{P}<0.05)$. Compared with ET biofilms, the overall constituent ratio of microflora was significantly different in throat swab and tracheal aspirate samples $(\mathrm{P}<0.05)$. However tracheal aspirate samples were useful for predicting Staphylococcus sp. in ET biofilms with a sensitivity of $85.7 \%$ and a specificity of $83.3 \%$. The sensitivity for the combination of tracheal aspirate and throat swab samples to detect Staphylococcus sp. in ET biofilms was $100 \%$. The detection of Pseudomonas sp. in throat swabs assisted its identification in ET biofilms (sensitivity $33.3 \%$ and specificity $100 \%$ ). The results of the present study suggest that microbial investigations in throat swab and tracheal aspirate samples are beneficial for identifying the ET biofilm flora. There may therefore be clinical applications of using substituent samples to identify pathogens in ET biofilms for VAP surveillance among intubated neonates.

\section{Introduction}

Ventilator-associated pneumonia (VAP) is a common complication among mechanical ventilated neonates in neonatal intensive care unit (NICU). It has been reported that VAP is the second most frequent nosocomial infection among patients in the pediatric intensive care unit (PICU) (1), and is the leading cause of death among ventilated patients (2). The incidence of VAP in NICU is even higher than that in PICU (3). A previous meta-analysis on neonatal VAP in China reported that the total weighted incidence and case fatality rates of VAP in NICU were $42.8 \%$ (95\% confidence interval; 39.8-45.9\%) and $16.4 \%$ (95\% confidence interval; $13.8-18.9 \%$ ), respectively (4). In spite of the high mobility and mortality, studies addressing neonatal VAP are limited in the literature (3). Thus, VAP in NICU remains a serious public health issue that endangers the lives of critically ill neonates.

Within $24 \mathrm{~h}$ following intubation, a polymicrobial biofilm harboring potential pathogens forms on the inner surface of the endotracheal tube (ET) (5-7). Bacterial biofilm cells are 
able to detach from the surface of the ET and travel to the lower airway due to gas flow during continuous ventilation (5), thus promoting VAP microbial persistence and consequently affecting patient prognosis $(7,8)$. Therefore, biofilms are crucial in the pathogenesis of VAP and a better understanding of biofilms, especially the biofilm flora, is required. However, studies on ET biofilm flora among intubated neonates are scarce. Furthermore, due to concerns for patient safety and the fact that reintubation itself carries a significant risk of neonatal VAP (3), ETs are not able to be extubated during intubation. The majority of ET samples are only available at the time of extubation (9-11), which means that the microbial signatures of ET biofilms prior to extubation are unknown. Therefore, identifying substituent samples to investigate microbiota in ET biofilms is beneficial to identify the floral risk factors associated with VAP. Specimens, such as throat swabs and tracheal aspirates, are able to be obtained at different times during intubation. Furthermore, it has been reported that a vast range of identical and potential pathogens are found in upper and lower respiratory tract $(12,13)$, and ET is the conduit between them. This indicates a potential consistence of microorganisms among throat swab, tracheal aspirate and ET biofilms, which means that tracheal aspirates and throat swabs may be the substituents of ET biofilms. A previous study demonstrated that nose swabs, throat swabs and sputum samples were able to predict the presence of several nosocomial pathogens in ET biofilms among intubated adults (14). However, this remains to be investigated in other populations, in particular intubated neonates. Furthermore, the previous study only addressed several nosocomial pathogens based on culture results (14). Culture dependent methods typically fail to recover and grow all biofilm cells from implanted medical devices, and therefore lack sensitivity for the detection of microflora $(10,15)$. Culture-independent methods based on microbial 16S ribosomal RNA (rRNA) genes overcome the limitation of culture, and allow a more comprehensive understanding of human microbiome (16). Denaturing gradient gel electrophoresis (DGGE) is a culture-independent method that facilitates rapid analyses and comparisons of microbial communities (17), and is simpler and less expensive than other culture-independent methods. DGGE has been widely applied in microbiological studies $(9,17,18)$, such as a recent study among intubated adults, which reported a considerable biofilm compositional complexity based on DGGE (9). However, to the best of our knowledge similar studies among neonatal patients are scarce, particularly those with VAP.

The present study utilized polymerase chain reaction (PCR)-DGGE to characterize the microbial communities in ET biofilms, throat swabs and tracheal aspirate samples of intubated neonates with VAP. The aim of the present study was to test the consistency of microbial communities among the three sources of specimens based on a culture-independent approach, thereby identifying alternatives for the surveillance of microbial profiles and potential pathogens in ET biofilms during intubation of intubated neonates.

\section{Materials and methods}

Patients. The study period ran from January to December 2014. A total of 15 mechanically ventilated neonates with VAP at the Children's Hospital of Chongqing Medical University (Chongqing, China) were included in the present study. VAP was diagnosed according to the accepted VAP criterion defined by the Centers for Disease Control and Prevention of America $(19,20)$. Of the 15 intubated neonates with VAP included in the present study, $8(53.3 \%)$ were male and $7(46.7 \%)$ were female (Table I). The gestational age of the study patients ranged from 28.6-41.3 weeks, including 10 $(66.7 \%)$ premature infants. A total of $8(53.3 \%)$ of the study patients were diagnosed with neonatal respiratory distress syndrome, followed by asphyxia neonatorum (20.0\%; Table I). The length of intubation ranged from 2-10.9 days, with a median of 5.8 days (Table I). Prior to extubation, antibiotics (moxalactam) were administered among all the study patients for a range of 1-13 days, with a median of 5.4 days (Table I).

Study samples. The ET was collected immediately following extubation and stored in sterile PBS at $4^{\circ} \mathrm{C}$. Vortex and sonication were conducted to release biofilms from the inner surface of the endotracheal tube as previously described (9). Prior to extubation, throat swabs and tracheal aspirates were collected and processed as previously described (14). The three types of specimen from each patient were collected and processed on the same day. Microbial DNA was extracted using a Mini BEST Bacterial Genomic DNA Extraction Kit Ver 3.0 (Takara Biotechnology Co., Ltd., Dalian, China) according to the manufacturer's protocol. The study protocol was reviewed and approved by the Medical Ethics Committee of Chongqing Medical University (099/2014). Informed consent was obtained from the parents or legal guardians of the study patients. Demographic and clinical data were obtained from medical records and anonymized information was used in the present study.

PCR-DGGE. The viable V3 region of $16 \mathrm{~S}$ rRNA gene was amplified using universal bacterial primers $357 \mathrm{f}$ with ' $\mathrm{GC}$ ' clamp (5'-CGCCCGGGGCGCGCCCCGGGCGGGGCG GGGGCAGGGGCCTACGGGAGGCAGCAG-3') and 518r (5'-ATTACCGCGGCTGCTGG-3'). The PCR was conducted as previously described (21). The reaction included $6 \mu 1$ microbial DNA as template DNA, $25 \mu$ 1 Premix Taq Version 2.0 (TaKaRa Biotechnology Co., Ltd., Dalian, China), $0.5 \mu$ l each primer and $18 \mu \mathrm{l}$ sterile $\mathrm{ddH}_{2} \mathrm{O}$. Template DNA of sterile $\mathrm{ddH}_{2} \mathrm{O}$ was regarded as the negative control. The reaction conditions were as follows: Initial denaturation at $94^{\circ} \mathrm{C}$ for $5 \mathrm{~min}, 10$ cycles of denaturation at $94^{\circ} \mathrm{C}$ for $30 \mathrm{sec}$, annealing at 61 to $56^{\circ} \mathrm{C}\left(-0.5^{\circ} \mathrm{C} /\right.$ cycle $)$, and extension at $72^{\circ} \mathrm{C}$ for $1 \mathrm{~min}$, 25 cycles of denaturation at $94^{\circ} \mathrm{C}$ for $30 \mathrm{sec}$, annealing at $56^{\circ} \mathrm{C}$ for $30 \mathrm{sec}$, and extension at $72^{\circ} \mathrm{C}$ for $1 \mathrm{~min}$, and a final extension at $72^{\circ} \mathrm{C}$ for $7 \mathrm{~min}$ (21). To evaluate the accuracy of PCR, the size of amplicons was determined by $2 \%$ agarose electrophoresis and visualized using Benchtop 3UV ultraviolet transilluminator (Ultra-Violet Products, Ltd., Cambridge, UK). The $195 \mathrm{bp}$ PCR products were processed for DGGE based on DCode Universal Mutation Detection System (Bio-Rad Laboratories, Inc., Hercules, CA, USA). Following $16 \mathrm{~h}$ of electrophoresis at $85 \mathrm{~V}$ and $60^{\circ} \mathrm{C}$, the DGGE gels were stained using SYBR-Green I (Bioteke Corporation, Beijing, China) for 30 min and scanned using Benchtop 3UV ultraviolet transilluminator (UVP, Inc., Upland, CA, USA). Visible bands in the 


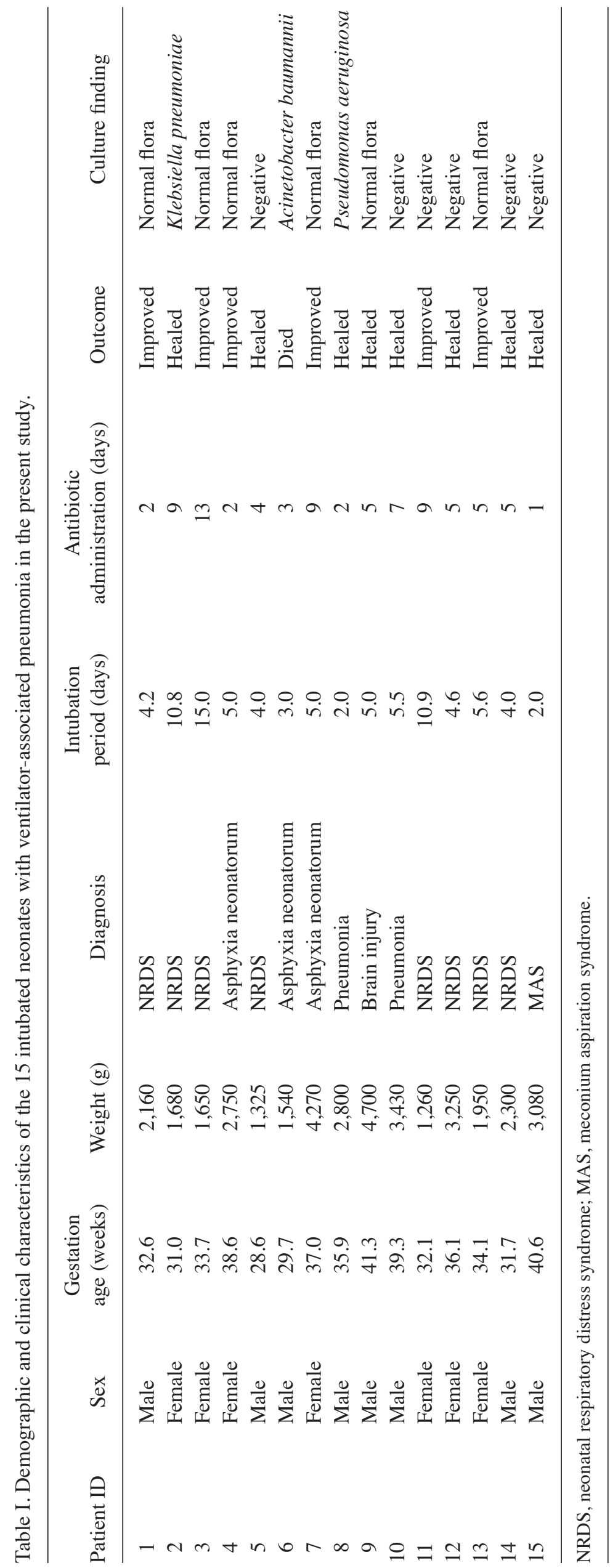


DGGE gel were excised, washed twice with sterile $\mathrm{ddH}_{2} \mathrm{O}$, placed in $30 \mu \mathrm{l}$ sterile $\mathrm{ddH}_{2} \mathrm{O}$ and incubated at $4^{\circ} \mathrm{Covernight}$ to release DNA.

Cloning and sequencing. A second PCR targeting 16S rRNA gene $\mathrm{V} 3$ region was conducted using primers $357 \mathrm{f}$ without the 'GC' clamp (5'-CCTACGGGAGGCAGCAG-3') and 518r as previously described (21). The PCR products were purified using Agarose Gel DNA Purification Kit Version 3.0 (Takara Bio, Inc., Otsu, Japan). The purified DNA was cloned using the PMD18-T Vector system (Takara Bio,Inc.) and transformed into Escherichia coli DH5a competent cells (Takara Biotechnology Co., Ltd.). The resulting cells were cultured overnight at $37^{\circ} \mathrm{C}$ on Luria-Bertani (LB) medium with ampicillin and positive clones were selected for sequencing (Biotech Corporation, Shanghai, China) as described previously (21). Sequences were assigned and identified to phylogenetic species using BLAST in NCBI Genbank database (https://blast.ncbi.nlm. nih.gov/Blast.cgi) with 97\% similarity. The PCR experiments performed were repeated twice.

Diversity and similarity analysis of DGGE fingerprints. DGGE profiles were analyzed and compared using Quantity One software (Version 4.6.2; Bio-Rad Laboratories, Inc.). To evaluate the microbial diversity and richness, the Shannon-Wiener index and Simpson's index for each sample were calculated using Bio-Dap software (nhsbig.inhs.uiuc. edu/wes/populations.html). The band number of each sample in DGGE images was also analyzed to evaluate the microbial diversity. Cluster maps of DGGE fingerprints were generated based on the Dice coefficient using the unweighted pair group method with arithmetic averages (UPGMA) to assess the similarity between samples in each DGGE image using Quantity-One software.

Statistical analysis. To assess the consistency of microflora in endotracheal tube biofilms with throat swab and tracheal aspirate, microbial diversity and taxonomic composition were compared using independent sample t-test, Chi-square test and Fisher's exact test as appropriate. Sensitivities and specificities for alternative samples (i.e., throat swab and tracheal aspirate samples) for the detection of certain taxa in ET biofilms were also calculated based on the microbiological findings in ET biofilms. All the statistical analyses were performed using SPSS 18.0 (SPSS, Inc., Chicago, IL, USA). P $<0.05$ was considered to indicate a statistically significant difference.

\section{Results}

A total of $8(53.3 \%)$ of the study patients were healed, clinical symptoms of $6(40.0 \%)$ patients were improved and the remaining patient succumbed to mortality. Clinical culture results of tracheal aspirate (following extubation) demonstrated that $60.0 \%$ of patients were culture-positive. Among those culture-positive subjects, normal flora was the most frequently identified, accounting for $66.7 \%$ (6/9; Table I).

Microbial diversity analysis in the study samples. PCR-DGGE was successfully performed for all 15 throat swabs and ET biofilm samples; however $2(13.3 \%)$ tracheal aspirate samples
Table II. Sequencing results of denaturing gradient gel electrophoresis bands.

\begin{tabular}{llc}
\hline $\begin{array}{l}\text { NCBI BLAST } \\
\text { result }\end{array}$ & $\begin{array}{c}\text { Accession } \\
\text { number }\end{array}$ & Identity (\%) \\
\hline Acinetobacter $s p$. & KR088390.1 & 99 \\
Bacillus $s$. & AJ000648.1 & 99 \\
Burkholderia $s p$. & AB011288.1 & 98 \\
Corynebacterium $s p$. & AB012207.1 & 98 \\
Klebsiella sp. & KP645384.1 & 100 \\
Pseudomonas $s p$. & HQ268805.1 & 99 \\
Rothia sp. & KC632201.1 & 99 \\
Staphylococcus $s p$. & KT277495.1 & 100 \\
Streptococcus $s p$. & KM225751.1 & 100 \\
Ureaplasma $s p$. & AF073457.1 & 100 \\
\hline
\end{tabular}

failed to generate a positive PCR result. The DGGE fingerprints for the three types of specimens are displayed in Fig. 1. No significant difference between the three sources of specimens was found in the Dice coefficients in UPGMA based cluster maps (Fig. 1), with no significant differences in the microbial similarity among the three types of samples. Shannon-Wiener index, Simpson's index and band number of tracheal aspirate samples were similar to those of ET biofilms (Fig. 2), which indicates a similar level of microbial diversity between the ET biofilm and tracheal aspirate samples. However, significant differences were found between throat swab and ET biofilm samples in the above three indices $(\mathrm{P}<0.05$; Fig. 2$)$.

Taxonomic analysis of microbial community in the study samples. A total of 10 genera were identified in the study samples (Table II). The overall constituent ratio of microflora differed significantly in throat swabs and tracheal aspirates compared with ET biofilm samples ( $\mathrm{P}<0.05$; Fig. 3). Notably, four genera (Pseudomonas sp., Staphylococcus sp., Streptococcus sp. and Burkholderia sp.) were shared by the three different types of specimens. Klebsiella $s p$. was identified in ET biofilm and tracheal aspirate. As a common pathogen to cause VAP (4), Klebsiella sp. was also included in the subsequent analysis. To evaluate the consistency of the detection of identical taxa, the detection rates of the five common genera in throat swab and tracheal aspirate were compared with ET biofilms (Table III). The detection rates of Staphylococcus sp. and Streptococcus sp. were similar between ET biofilms and the corresponding tracheal aspirate samples (Table III); however, a significant difference was observed in the detection rate of Pseudomonas sp. between ET biofilms and the corresponding tracheal aspirate samples $(\mathrm{P}<0.05$; Table III). The detection rates of Pseudomonas sp. and Streptococcus sp. in throat swab samples were significantly different from those in ET biofilm samples $(\mathrm{P}<0.05$; Table III $)$.

Sensitivity and specificity for the detection of ET biofilm flora. To assess the possibility of using throat swabs, tracheal aspirates and a combination of throat swab and tracheal aspirate samples to predict microbial components in ET biofilms, 

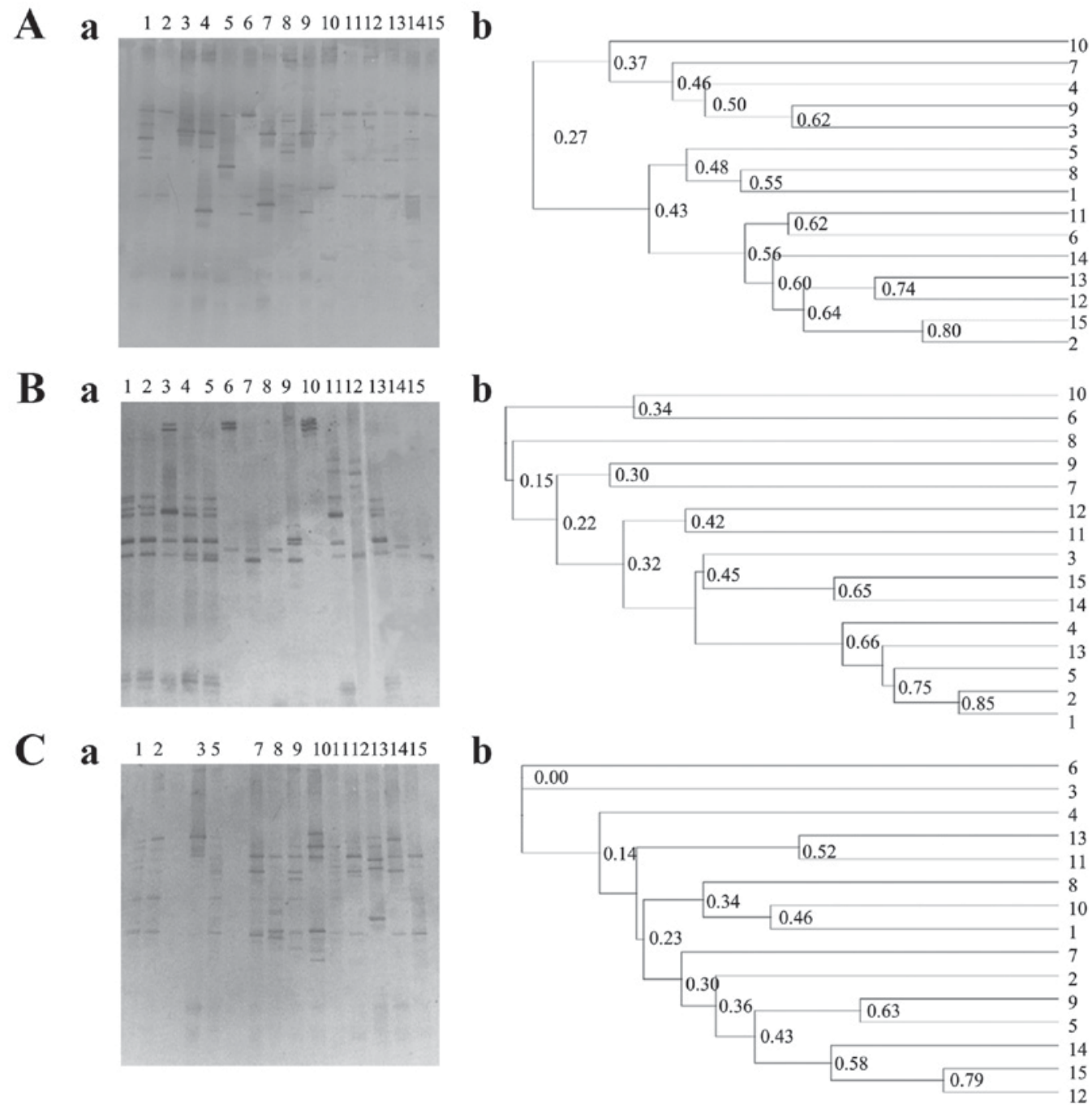

Figure 1. DGGE fingerprints and cluster maps of the (A) throat swab, (B) endotracheal tube biofilm and (C) tracheal aspirate samples from 15 patients. (a) Shows the DGGE fingerprints and (b) shows its corresponding cluster maps. Dice coefficients were shown in the clusters maps (b) to assess the similarities between samples. DGGE, denaturing gradient gel electrophoresis.

their sensitivities and specificities were calculated to detect the five genera in ET biofilms (Table IV). For the detection of Staphylococcus sp. in ET biofilms, the sensitivity for tracheal aspirate samples was $85.7 \%$, which was higher than that for throat swab samples (37.5\%; Table IV). Furthermore, the sensitivity for the combination of tracheal aspirate and throat swab samples for Staphylococcus sp. detection in ET biofilms was $100 \%$ (Table IV). Although the sensitivity of throat swab samples for detecting Pseudomonas $s p$. in ET biofilms was $33.3 \%$, the specificity was $100 \%$ (Table IV). The sensitivities to detect Klebsiella $s p$. were low in all the three groups (i.e., throat swab, tracheal aspirate and combined throat swab and tracheal aspirate groups; Table IV). The sensitivities to detect Streptococcus $s p$. in all the three groups were high; however the corresponding specificities were low (Table IV).

\section{Discussion}

ET biofilm is an important source in facilitating bacterial contamination of the lower airway $(10,22)$ and so is critical for VAP surveillance. To the best of our knowledge, this is the first study to address the consistency of microbial signatures in ET biofilms and the corresponding throat swab and tracheal aspirate samples using a culture-independent approach among newborns. There were four major findings of the present study. Firstly, the microbial richness and diversity of ET biofilms were similar to tracheal aspirate samples, however significantly different from throat swab samples. Secondly, the taxa composition varied significantly among the three sources of specimens. Thirdly, tracheal aspirate samples performed well in the detection of Staphylococcus sp. in ET biofilms, with a sensitivity of $85.7 \%$ and a specificity of $83.3 \%$. Finally, the detection of Pseudomonas $s p$. in throat swabs helped its identification in ET biofilms (sensitivity $33.3 \%$ and specificity $100 \%)$.

A previous study has demonstrated that decreased microbial diversity may be associated with VAP among intubated neonates (21), indicating that it is an important measurement for VAP surveillance. In the present study, the findings of similar microbial diversity shared by tracheal aspirate and ET biofilm samples suggests that the microbial diversity profile of ET biofilms may be evaluated using the corresponding tracheal aspirate samples instead. However, throat swab samples harbored a significantly higher microbial diversity 
Table III. Detection rates of the five common genera among throat swab, ET biofilm and tracheal aspirate specimens respectively in the present study.

\begin{tabular}{|c|c|c|c|c|c|}
\hline Genus & $\begin{array}{c}\text { Throat swab, } \\
\text { detection rate }(\%)\end{array}$ & $\begin{array}{c}\text { ET biofilm, } \\
\text { detection rate }(\%)\end{array}$ & P-value & $\begin{array}{l}\text { Tracheal aspirate, } \\
\text { detection rate }(\%)\end{array}$ & P-value ${ }^{b}$ \\
\hline Staphylococcus & $5 / 15(33.3)$ & $8 / 15(53.3)$ & 0.27 & $7 / 13(53.8)$ & 0.98 \\
\hline Klebsiella & $0 / 15(0.0)$ & $7 / 15(46.7)$ & $0.06^{\mathrm{c}}$ & $3 / 13(23.1)$ & $0.25^{\mathrm{c}}$ \\
\hline Pseudomonas & $4 / 15(26.7)$ & $12 / 15(80.0)$ & $<0.01$ & $1 / 13(7.7)$ & $<0.01$ \\
\hline Burkholderia & $6 / 15(40.0)$ & $7 / 15$ (46.7) & 0.71 & $8 / 13(61.5)$ & 0.43 \\
\hline Streptococcus & $15 / 15(100.0)$ & 9/15 (60.0) & $0.02^{\mathrm{c}}$ & $8 / 13(61.5)$ & 0.93 \\
\hline
\end{tabular}

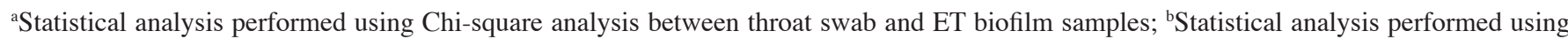
Chi-square analysis between tracheal aspirate and ET biofilm samples; ${ }^{c}$ Statistical analysis performed using Fisher exact test. ET, endotracheal tube.
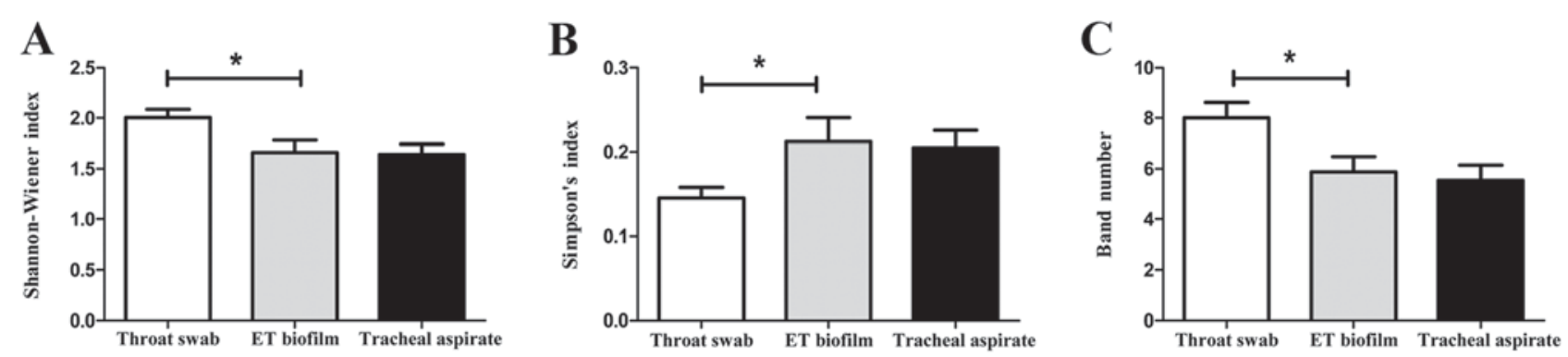

Figure 2. Comparisons of Shannon-Wiener index, Simpson's index and band number of throat swab, ET biofilm and tracheal aspirate samples. Significant differences were observed in (A) Shannon-Wiener index, (B) Simpson's index and (C) band number between throat swab and ET biofilm samples. "P<0.05 vs. ET biofilm. ET, endotracheal tube.

and richness than ET biofilms, which indicates that they are not suitable as a substitute to represent the microbial diversity and richness of ET biofilm samples.

In the present study, the presence of Staphylococcus sp. was found to be similarly distributed in tracheal aspirate samples and ET biofilms. Furthermore, the sensitivity and specificity to detect Staphylococcus sp. in ET biofilms by tracheal aspirate samples were high (85.7 and $83.3 \%$, respectively, Table IV). It is well known that common VAP pathogen Staphylococcus aureus and nosocomial pathogen Staphylococcus epidermidis are important members of Staphylococcus sp., especially Staphylococcus aureus (4). A review based on the results of the SENTRY Antimicrobial Surveillance Program (1997-2008, 31,436 cases) reported that Staphylococcus aureus was the most common causative pathogen (28\%) in VAP (22). Therefore, the detection of Staphylococcus $s p$. is important and may be useful for VAP diagnosis and treatment. The findings of the present study suggest that the detection of Staphylococcus $s p$. in tracheal aspirate may predict its presence in ET biofilms among intubated neonates.

Previous studies have reported a high concordance of bacterial culture results between tracheal aspirate samples and ET biofilms $(8,23)$. For example, in a previous study conducted among adults undergoing mechanical ventilation in Spain, identical microorganisms with ET biofilms were isolated in $56 \%$ of tracheal aspirate samples (8). However, based on a culture-independent approach, the results of the present study demonstrated that the overall microbial composition was different in tracheal aspirates compared with ET biofilm specimens in neonates with VAP, with a significant difference in the presence of Pseudomonas sp. between tracheal aspirate and ET biofilm samples (Table III). This suggests that microflora in tracheal aspirate samples may not entirely represent all the microbial components correctly in ET biofilms. Pseudomonas $s p$. is considered to be a common and important nosocomial pathogen to cause VAP (4). The findings of the present study suggest that the presence of Pseudomonas sp. in tracheal aspirate has little predictive value of its presence in ET biofilms. This may possibly be due to the distinct difference in responses to systemic antibiotics between planktonic and biofilm microorganisms. Bacteria in tracheal aspirates are present as a planktonic form, which are exposed to antibiotics and more likely to be killed. However, bacteria in ET biofilms are considered to be drug-resistant and free from systemic antibiotics $(8,24)$, which may lead to the disparity in the levels of Pseudomonas sp. in the two different types of samples. The similar constituent ratio of Staphylococcus $s p$. between tracheal aspirate and ET biofilms suggests the presence of drug-resistant Staphylococcus sp. strains in tracheal aspirate in the study samples. This is consistent with several epidemiological studies conducted in China demonstrating the relatively high drug-resistant rate of Staphylococcus $s p$. strains in NICU $(4,25)$. 
Table IV. Sensitivity and specificity to detect the five major genera in endotracheal tube biofilms for the corresponding throat swab, tracheal aspirate and the combination of throat swab and tracheal aspirate samples, respectively.

\begin{tabular}{|c|c|c|c|c|c|c|}
\hline \multirow[b]{2}{*}{ Genus } & \multicolumn{3}{|c|}{ Sensitivity (\%) } & \multicolumn{3}{|c|}{ Specificity (\%) } \\
\hline & $\mathrm{T}$ & A & $\mathrm{T}+\mathrm{A}$ & $\mathrm{T}$ & A & $\mathrm{T}+\mathrm{A}$ \\
\hline Staphylococcus & $37.5(3 / 8)$ & $85.7(6 / 7)$ & $100.0(8 / 8)$ & $71.4(5 / 7)$ & $83.3(6 / 6)$ & $71.4(5 / 7)$ \\
\hline Klebsiella & $0.0(0 / 7)$ & $28.6(2 / 7)$ & $28.6(2 / 7)$ & $100.0(8 / 8)$ & $87.5(7 / 8)$ & $87.5(7 / 8)$ \\
\hline Pseudomonas & $33.3(4 / 12)$ & $9.1(1 / 11)$ & $33.3(4 / 12)$ & $100.0(3 / 3)$ & $100.0(2 / 2)$ & $100.0(3 / 3)$ \\
\hline Burkholderia & $57.1(4 / 7)$ & $50.0(3 / 6)$ & $85.7(6 / 7)$ & $75.0(6 / 8)$ & $28.6(2 / 7)$ & $37.5(3 / 8)$ \\
\hline Streptococcus & $100.0(9 / 9)$ & $62.5(5 / 8)$ & $100.0(9 / 9)$ & $0.0(0 / 6)$ & $40.0(2 / 5)$ & $0.0(0 / 6)$ \\
\hline
\end{tabular}

T, throat; A, aspirate.

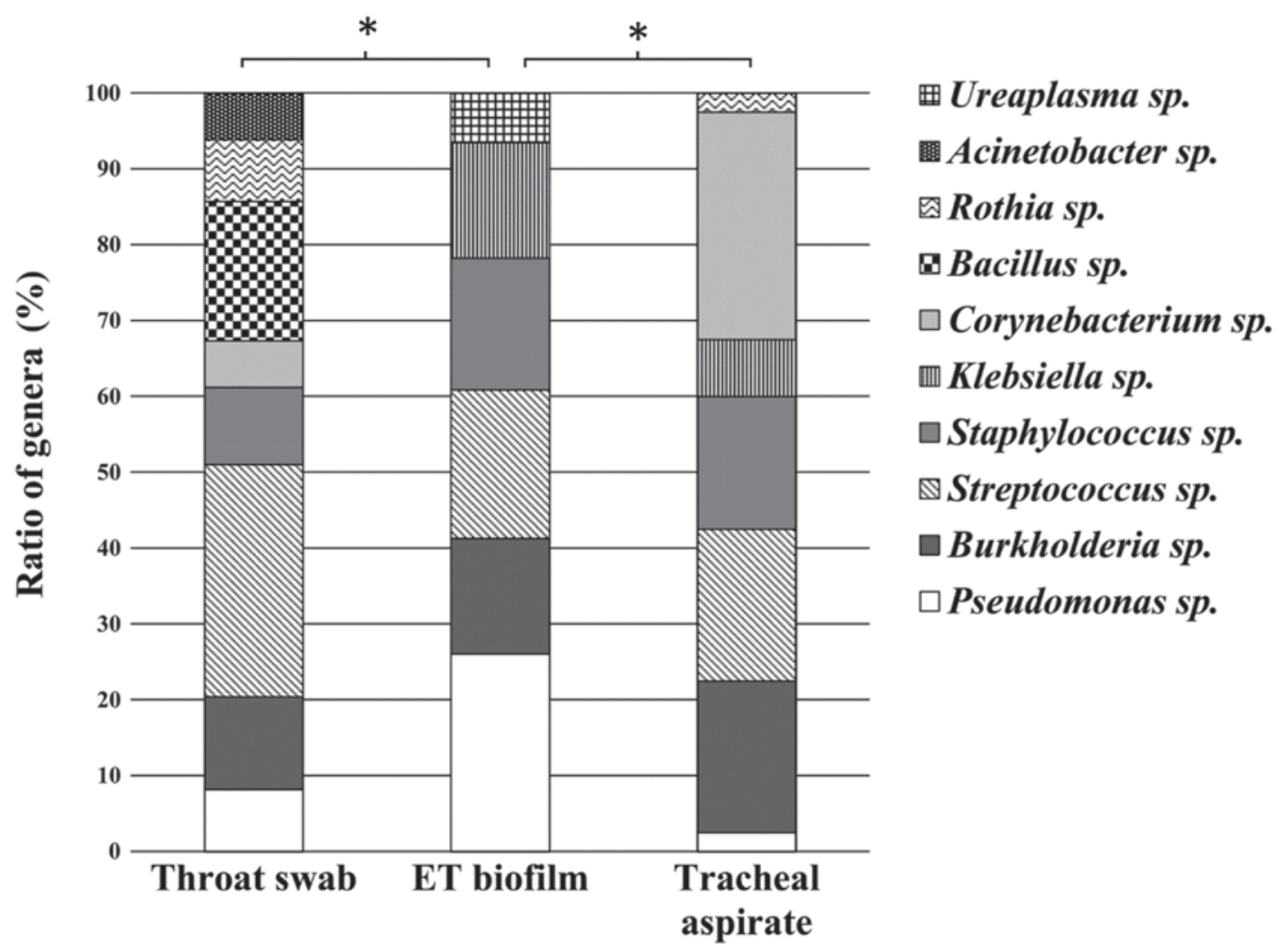

Figure 3. Composition ratio of the genera among throat swab, ET biofilm and tracheal aspirate samples. Significant differences were shown in the composition ratio of the genera between ET biofilm and throat swab, and between ET biofilm and tracheal aspirate samples. ${ }^{*} \mathrm{P}<0.05$ vs. ET biofilm. ET, endotracheal tube.

Unlike healthy patients (26), gram-negative bacilli such as Pseudomonas aeruginosa are typically observed in the oropharynx of critically ill hospitalized patients $(22,27)$. These bacilli are gram-negative and are considered to be associated with the onset of nosocomial pneumonia (28). Although a statistical difference was observed in the constitute ratio of Pseudomonas sp. between throat swab and ET biofilm samples in the present study, it was also noted that of the 4 patients with Pseudomonas sp. present in throat swab, Pseudomonas sp. was also identified in the corresponding ET biofilms, with a specificity of $100 \%$. This suggests that throat swabs may have a potential role in facilitating the detection of Pseudomonas sp. in ET biofilms. Feldman et al (23) reported the time sequence of bacterial colonization in VAP patients, and demonstrated that potential pathogens first colonized the oropharynx and then the lower respiratory tract. Therefore, there may be a high consistency of bacteria between oropharynx and ET biofilms in the early stages of VAP. To confirm this, the study isolates were classified into early-onset VAP (intubated for $<5$ days) and late-onset VAP groups (intubated for $\geq 5$ days), and found the sensitivity for throat swab samples to detect Pseudomonas sp. in ET biofilms was higher among early-onset VAP (60.0\%) subjects than the late-onset subjects (14.3\%). Therefore, the detection of Pseudomonas sp. in throat swabs may be helpful to predict its presence in ET biofilms of intubated neonates, particularly for early-onset VAP patients. 
Significant differences were observed in the detection of Streptococcus sp. between throat swab and ET biofilm samples in the present study $(\mathrm{P}<0.05)$. These results suggest that throat swab samples are not useful for the identification of Streptococcus sp in ET biofilms. As the common colonizers in the oropharynx, Streptococcus sp. is dominant in the oropharynx among infants (29). The results of the present study confirmed this, with the detection rate of Streptococcus sp. being $100.0 \%$ in throat swab samples. Simultaneously, the prevalence of Streptococcus $s p$. in ET biofilms was found to be $60.0 \%$, which suggests Streptococcus sp. in ET biofilms may originate from the oropharynx. It has been reported that Streptococcus sp. enhanced biofilm formation of other nosocomial pathogens such as Pseudomonas aeruginosa (30), which is associated with the progression of VAP. The results of the present study indicate the possible source of Streptococcus sp. in ET biofilms among intubated neonates and suggest the need to develop strategies against Streptococcus sp. in the oropharynx to prevent VAP among intubated neonates.

It has been reported that combined culture results of throat swab, nose swab and sputum increases the sensitivity to detect nosocomial pathogens in ET biofilms compared with results from a single sample in intubated adults (14). In the present study, the performance of a combination of throat swabs and tracheal aspirates was assessed to identify potential pathogens in ET biofilms (Table IV). The combination of samples indeed raised the sensitivity, however, decreased the specificity, which impedes its further application.

The present study has some limitations. Firstly, the samples size of the present study is small; however, it provides the preliminary microbial data for neonatal VAP based on the comprehensive culture-independent method. These findings need to be confirmed and generalized using a larger sample size of intubated neonates. Secondly, due to the limited resolution of the 16S rRNA gene V3 region, the resulting sequences are only able to be identified and assigned to the genus level. As a result, further taxonomic information at species level is not provided.

In conclusion, the present study extended the knowledge base of the microbial characteristics of intubated neonates with VAP. The results suggest that microbial investigations in throat swab and tracheal aspirate samples are beneficial to investigate microbial profile and identify potential pathogens in ET biofilms among intubated neonates, and suggest that there are potential applications of using substituent samples to investigate microbial signatures in ET biofilms for VAP surveillance in NICU.

\section{Acknowledgements}

The authors of the present study would like to thank Mrs. Xianhong Zhang in the Department of Neonatology, Children's Hospital of Chongqing Medical University for her excellent assistance in sample collection. This work was supported by the National Natural Science Foundation of China (grant no. 81370744), the Doctoral Degree Funding from Chinese Ministry of Education (grant no. 20135503110009), the State Key Clinic Discipline Project (grant no. 2011-873), the Subproject of National Science \& Technology Pillar Program during the 12th Five-year Plan Period in China (grant no. 2012BAI04B05), and the Clinical Research Program of Children's Hospital of Chongqing Medical University (grant no. lcyj2014-11).

\section{References}

1. National Nosocomial Infections Surveillance System: National Nosocomial Infections Surveillance (NNIS) System Report, data summary from January 1992 through June 2004, issued October 2004. Am J Infect Control 32: 470-485, 2004.

2. Aly H, Badawy M, El-Kholy A, Nabil R and Mohamed A: Randomized, controlled trial on tracheal colonization of ventilated infants: Can gravity prevent ventilator-associated pneumonia? Pediatrics 122: 770-774, 2008.

3. Tan B, Zhang F, Zhang X, Huang YL, Gao YS, Liu X, Li YL and Qiu JF: Risk factors for ventilator-associated pneumonia in the neonatal intensive care unit: A meta-analysis of observational studies. Eur J Pediatr 173: 427-434, 2014.

4. Tan B, Xian-Yang X, Zhang X, Peng-Zhou X, Wang P, Xue J, Ling-Huang Y, Li-Li Y and Fu-Qiu J: Epidemiology of pathogens and drug resistance of ventilator-associated pneumonia in Chinese neonatal intensive care units: A meta-analysis. Am J Infect Control 42: 902-910, 2014.

5. Inglis T, Millar M, Jones $\mathrm{J}$ and Robinson D: Tracheal tube biofilm as a source of bacterial colonization of the lung. J Clin Microbiol 27: 2014-2018, 1989.

6. Adair C, Gorman S, Feron B, Byers L, Jones D, Goldsmith C, Moore J, Kerr J, Curran M, Hogg G, et al: Implications of endotracheal tube biofilm for ventilator-associated pneumonia. Intensive Care Med 25: 1072-1076, 1999.

7. Sottile FD, Marrie TJ, Prough DS, Hobgood CD, Gower DJ, Webb LX, Costerton JW and Gristina AG: Nosocomial pulmonary infection: Possible etiologic significance of bacterial adhesion to endotracheal tubes. Crit Care Med 14: 265-270, 1986.

8. Gil-Perotin S, Ramirez P, Marti V, Sahuquillo JM, Gonzalez E, Calleja I, Menendez R and Bonastre J: Implications of endotracheal tube biofilm in ventilator-associated pneumonia response: A state of concept. Crit Care 16: R93, 2012.

9. Cairns S, Thomas JG, Hooper SJ, Wise MP, Frost PJ, Wilson MJ, Lewis MA and Williams DW: Molecular analysis of microbial communities in endotracheal tube biofilms. PLoS One 6: e14759, 2011.

10. Vandecandelaere I, Matthijs N, Van Nieuwerburgh F, Deforce D, Vosters P, De Bus L, Nelis HJ, Depuydt P and Coenye T: Assessment of microbial diversity in biofilms recovered from endotracheal tubes using culture dependent and independent approaches. PLoS One 7: e38401, 2012.

11. Perkins SD, Woeltje KF and Angenent LT: Endotracheal tube biofilm inoculation of oral flora and subsequent colonization of opportunistic pathogens. Int J Med Microbiol 300: 503-511, 2010.

12. Charlson ES, Bittinger K, Haas AR, Fitzgerald AS, Frank I, Yadav A, Bushman FD and Collman RG: Topographical continuity of bacterial populations in the healthy human respiratory tract. Am J Respir Crit Care Med 184: 957-963, 2011.

13. Pneumatikos IA, Dragoumanis CK and Bouros DE: Ventilatorassociated pneumonia or endotracheal tube-associated pneumonia? An approach to the pathogenesis and preventive strategies emphasizing the importance of endotracheal tube. Anesthesiology 110: 673-680, 2009.

14. Vandecandelaere I, Matthijs N, Nelis HJ, Depuydt P and Coenye $\mathrm{T}$ : The presence of antibiotic-resistant nosocomial pathogens in endotracheal tube biofilms and corresponding surveillance cultures. Pathog Dis 69: 142-148, 2013.

15. Costerton JW, Post JC, Ehrlich GD, Hu FZ, Kreft R, Nistico L, Kathju S, Stoodley P, Hall-Stoodley L, Maale G, et al: New methods for the detection of orthopedic and other biofilm infections. FEMS Immunol Med Microbiol 61: 133-140, 2011.

16. Wang Y, Hoenig JD, Malin KJ, Qamar S, Petrof EO, Sun J, Antonopoulos DA, Chang EB and Claud EC: 16S rRNA gene-based analysis of fecal microbiota from preterm infants with and without necrotizing enterocolitis. ISME J 3: 944-954, 2009.

17. Lu H, Qian G, Ren Z, Zhang C, Zhang H, Xu W, Ye P, Yang Y and Li L: Alterations of Bacteroides sp., Neisseria sp., Actinomyces $s p$. and Streptococcus sp. populations in the oropharyngeal microbiome are associated with liver cirrhosis and pneumonia. BMC Infect Dis 15: 239, 2015. 
18. Liu D, Yu J, Li L, Ai Q, Feng J, Song C and Li H: Bacterial community structure associated with elective cesarean section versus vaginal delivery in Chinese newborns. J Pediatr Gastroenterol Nutr 60: 240-246, 2015.

19. Centers for Disease Control and Prevention: Criteria for defining nosocomial pneumonia. http://www cdc gov/ncidod/hip/NNIS/ members/pneumonia/Final/PneumoCriteriaV1 pdf. Accessed February 5, 2009.

20. Cernada M, Brugada $M$, Golombek $S$ and Vento $M$ Ventilator-associated pneumonia in neonatal patients: An update. Neonatology 105: 98-107, 2014.

21. Lu W, Yu J, Ai Q, Liu D, Song C and Li L: Increased constituent ratios of Klebsiella sp., Acinetobacter sp. and Streptococcus sp. and a decrease in microflora diversity may be indicators of ventilator-associated pneumonia: A prospective study in the respiratory tracts of neonates. PLoS One 9: e87504, 2014.

22. Jones RN: Microbial etiologies of hospital-acquired bacterial pneumonia and ventilator-associated bacterial pneumonia. Clin Infect Dis 51 (Suppl 1): S81-S87, 2010.

23. Feldman C, Kassel M, Cantrell J, Kaka S, Morar R, Mahomed AG and Philips J: The presence and sequence of endotracheal tube colonization in patients undergoing mechanical ventilation. Eur Respir J 13: 546-551, 1999.
24. Wolcott RD and Ehrlich GD: Biofilms and chronic infections. Jama 299: 2682-2684, 2008.

25. Zhu XL, Zhao L, Yang JC, Chen X and Wu XH: Etiology and high risk factors of neonatal ventilator-associated pneumonia. Zhongguo Dang Dai Er Ke Za Zhi 9: 549-552, 2007 (In Chinese).

26. Safdar N, Crnich CJ and Maki DG: The pathogenesis of ventilator-associated pneumonia: Its relevance to developing effective strategies for prevention. Respir Care 50: 725-741, 2005.

27. Brennan MT, Bahrani-Mougeot F, Fox PC, Kennedy TP, Hopkins S, Boucher RC and Lockhart PB: The role of oral microbial colonization in ventilator-associated pneumonia. Oral Surg Oral Med Oral Pathol Oral Radiol Endod 98: 665-672, 2004.

28. Johanson WG Jr, Pierce AK, Sanford JP and Thomas GD: Nosocomial respiratory infections with gram-negative bacilli: The significance of colonization of the respiratory tract. Ann Intern Med 77: 701-706, 1972.

29. Rotimi V and Duerden B: The development of the bacterial flora in normal neonates. J Med Microbiol 14: 51-62, 1981.

30. Song S, Du L, Yu J, Ai Q, Pan Y, Fu Y and Wang Z: Does Streptococcus mitis, a neonatal oropharyngeal bacterium, influence the pathogenicity of Pseudomonas aeruginosa? Microbes Infect 17: 710-716, 2015. 